\title{
Hemiarthroplasty for Distal Humerus Fracture: A Systematic Review and Meta-analysis for Functional Outcome
}

\author{
Jae-Man Kwak ${ }^{1}$, Erica Kholinne ${ }^{1,2}$, Yucheng Sun ${ }^{1}$, Gwan Bum Lee ${ }^{1}$, Kyoung Hwan Koh ${ }^{1}$, Jae-Myeung Chun ${ }^{3}$, \\ In-Ho Jeon ${ }^{1 凶}$ \\ ${ }^{1}$ Department of Orthopedic Surgery, Asan Medical Center, University of Ulsan College of Medicine, Seoul, Korea, ${ }^{2}$ Department of Orthopedic Surgery, St. Carolus \\ Hospital, Jakarta, Indonesia, ${ }^{3}$ Department of Orthopaedic Surgery, Armed Forces Capital Hospital, Seongnam, Korea
}

Background: Treatment of distal humerus fractures in osteoporotic elderly patients is often challenging. For non-reconstructible fractures with open reduction and internal fixation, total elbow arthroplasty (TEA) is an acceptable alternative. However, the relatively high complication rates and lifelong activity restrictions make TEA less ideal for elderly or low-demand patients. Efforts to identify or develop alternate procedures that benefit relatively young, high-demand patients have resulted in increased interest in hemiarthroplasty. This systematic review reports the clinical outcomes of hemiarthroplasty for distal humeral fractures.

Methods: We systematically reviewed the databases of PubMed, Ovid MEDLINE, and Cochrane Library. All English-language studies published before June 2017 were considered for possible inclusion. Search terms included 'distal humerus fracture' and 'hemiarthroplasty'. Studies reporting outcomes (and a minimum of 1 year clinical follow-up) in human subjects after hemiarthroplasty (Latitude system) for distal humeral fractures were assessed for inclusion. Patient demographics, clinical and radiographic outcomes, and complications were recorded, and homogenous outcome measures were analyzed.

Results: Nine studies with a total of 115 patients met the inclusion criteria. Among the included studies, the weighted mean follow-up time was 35.4 months. Furthermore, the weighted mean of the postoperative range of motion ( $107.6^{\circ}$ flexion-extension, $157.5^{\circ}$ for pronation-supination) and functional outcomes (Mayo elbow performance scores: 85.8, Disabilities of the Arm, Shoulder and Hand score: 19.6) were within the acceptable range.

Conclusions: Our study indicates that hemiarthroplasty is a viable option for comminuted distal humerus fracture. Satisfactory functional outcomes were observed in most patients.

(Clin Shoulder Elbow 2018;21(3):120-126)

Key Words: Hemiarthroplasty; Humerus; Elbow; Fracture; Meta analysis

\section{Introduction}

Open reduction and internal fixation (ORIF) to restore the articular surface is the conventional treatment for distal humerus fractures. ${ }^{1-3)}$ However, it is sometimes impossible to restore articular congruency (for example, in cases with osteoporotic bones or severely comminuted fractures), making the conventional option more challenging and less effective. Since complications and reduction loss rates as high as 50\% have been reported after conventional ORIF for comminuted fractures, ${ }^{4)}$ arthroplasty has been advocated as a suitable alternative in such cases. Elbow arthroplasty has been practiced since 20 years, with continuous improvements in technique and device design. ${ }^{5)}$ An increasing number of studies have reported results related to geriatric distal humerus traumatology, and total elbow arthroplasty (TEA) is now considered an important treatment option. ${ }^{4,6,7)}$ While TEA offers

Received May 29, 2018. Accepted July 8, 2018.

Correspondence to: In-Ho Jeon

Department of Orthopedic Surgery, Asan Medical Center, University of Ulsan College of Medicine, 88 Olympic-ro 43-gil, Songpa-gu, Seoul 05505, Korea

Tel: +82-2-3010-3896, Fax: +82-2-488-7877, E-mail: jeonchoi@gmail.com, ORCID: https://orcid.org/0000-0002-9289-9193

This meta-analysis does not need an IRB approval document.

Financial support: None. Conflict of interests: None. 
an alternative option to treat comminuted distal humerus fractures, complications (including aseptic loosening, periprosthetic fracture, and infection) continue to limit the utility of this procedure in younger patients. Distal humerus hemiarthroplasty may therefore be more suitable for younger patients since it offers a shorter surgical time, does not generate polyethylene waste, and has a lower risk of component loosening as compared to TEA.

Mellen and Phalen ${ }^{8}$ first suggested the concept of hemiarthroplasty and made customized acrylic implants for young active soldiers with complex intra-articular fractures of the distal humerus at a US Army Hospital. The practice of hemiarthroplasty for complex distal humerus fractures has evolved since then. ${ }^{9-11)}$ While TEA is well described and reported, few studies have reported the outcomes of hemiarthroplasty for distal humerus fracture. Hence, there are no consensus guidelines for TEA indications or any reports on the outcomes to help elucidate age-dependent risks and benefits to guide management decisions. We found only one comparative systematic review of TEA versus distal humerus hemiarthroplasty, but the outcomes based on age and implant choice were not considered. ${ }^{12)}$ Thus, we aimed to analyze the clinical outcomes and complications associated with hemiarthroplasty using the single-implant system (the Latitude system) in distal humerus fractures through a systematic review of relevant literatures.

\section{Methods}

\section{Search Strategy}

The PRISMA (Preferred Reporting Items for Systematic Reviews and Meta-analyses) checklist and algorithm ${ }^{13)}$ guided this systematic review of literature found in the databases of PubMed, Ovid MEDLINE, and Cochrane Library. We combined the terms 'hemiarthroplasty' OR 'hemi prosthesis' OR 'hemi replacement' OR 'hemi' OR 'arthroplasty' AND 'distal humerus' OR 'distal humeral' OR 'distal humerus fracture' OR 'distal humeral fracture' OR 'elbow fracture'.

\section{Inclusion/Exclusion Criteria}

This review included studies of human subjects, with original data published in the English language that reported outcomes of hemiarthroplasty for distal humerus fractures, with a minimum follow-up of 1 year. Included studies were required to report clinical outcomes or complications after using the Latitude system for distal humerus fracture. Review articles and case reports were excluded.

\section{Data Extraction}

Data regarding study characteristics, patient demographics, indications and contraindications, inclusion and exclusion criteria, and outcome measures were extracted from each study that met the inclusion criteria. The literature search and data extrac- tion were performed by 4 blinded reviewers. Articles published on or before June 15, 2017 were considered. Only papers published in peer-reviewed journals were eligible, and relevance was established on the basis of article titles and abstracts. After article selection, bibliographies were assessed by the reviewers to identify further relevant articles. Three reviewers (GBL, EK, and JMK) reviewed each paper independently and discussed and resolved any disagreements on the inclusion or exclusion of any articles. The inclusion and exclusion of studies were also discussed with an expert orthopedic surgeon $(\mathrm{IHJ})$. Study characteristics of interest included author names, year of publication, study design, number of patients at the final follow-up, and length of follow-up. Patient demographics included sex and age. Outcomes of interest included postoperative functional outcome scores, radiographic assessment, complications, reoperations, and conversion to TEA. Descriptive statistics were used to report study characteristics, patient demographics, and outcomes.

\section{Quality Assessment}

The methodologic quality of the included studies was analyzed with the modified Coleman Methodology Score. ${ }^{14)}$ This score assesses the methodology of clinical studies using 10 specific quantitative and qualitative criteria: study size, mean follow-up, number of surgical procedures, type of study, diagnostic certainty, description of surgical procedure, postoperative rehabilitation, outcome measures, outcome assessment, and selection process. However, since this classification was originally designed for the knee, we partially modified the Coleman Methodology Score for appropriate assessment of the elbow. Potential scores range from 0 to 100, with a score of 100 indicating the highest study quality. The level of evidence of each study was determined using the Journal of Bone and Joint Surgery criteria.

\section{Statistical Analysis}

We also conducted a meta-analysis, for which the results using the random-effects model took into account the possible clinical diversity and methodological variations between studies. Specific analyses considering confounding factors were not possible due to unavailability of raw data. Categorical variables were expressed as frequencies with percentages, and continuous variables were expressed as mean values with ranges. Functional outcome and range of motion data were presented using standardized mean deviation calculations. All statistical analyses were performed with the IBM SPSS ver. 21.0 (IBM Co., Armonk, NY, USA). A pooling data analysis was performed using randomeffect models, which assumed that there were variations between studies. The chi-square tests studied the heterogeneity between trials. The $\mathrm{I}^{2}$ statistic was used to estimate the percentage of total variation across studies, owing to heterogeneity rather than chance, with values greater than $50 \%$ being considered as substantial heterogeneity. $I^{2}$ was calculated using the following 
formula: $I^{2}=100 \% \times(\mathrm{Q}-\mathrm{df}) / \mathrm{Q}$, where $\mathrm{Q}$ is defined as Cochrane's heterogeneity statistic and $\mathrm{df}$ is defined as degree of freedom. The possible clinical and methodological reasons in cases of substantial heterogeneity were qualitatively explored.

\section{Results}

Our literature search and references scan identified 231 relevant articles; of these, 218 were excluded for various reasons as

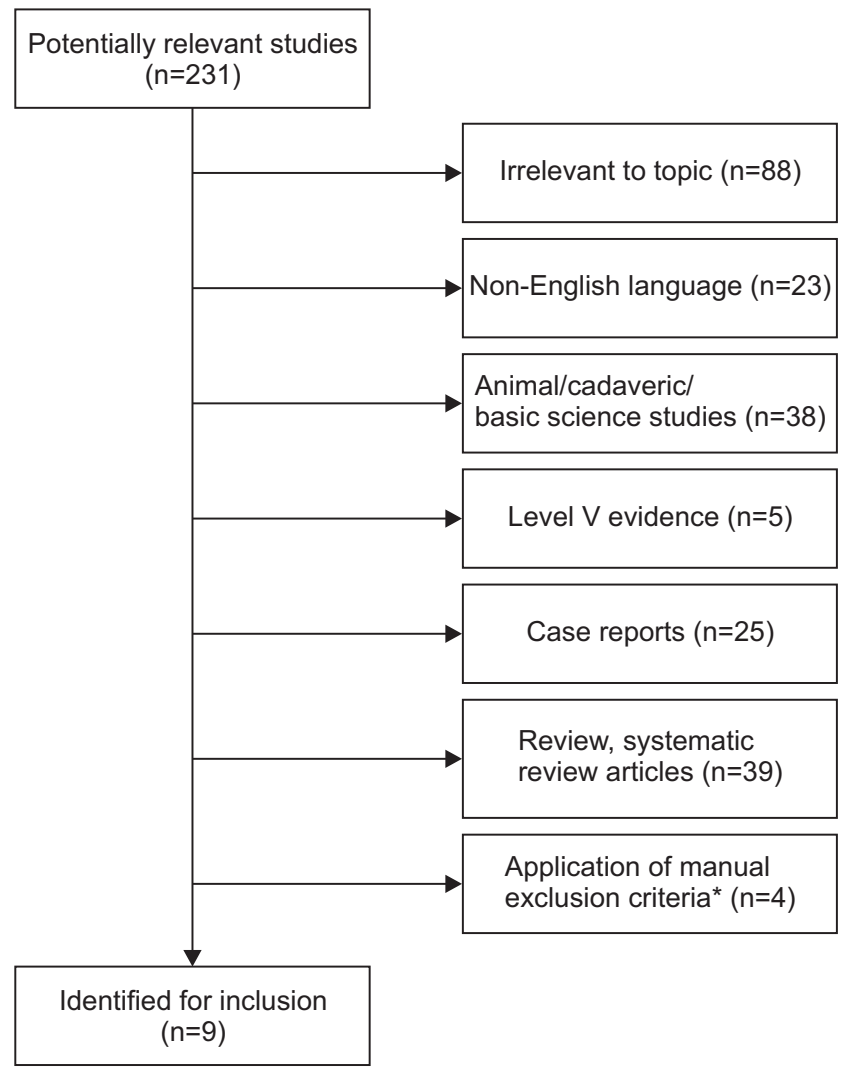

indicated (Fig. 1). Evaluation of the remaining articles excluded further 4 articles due to short follow-up durations (less than 1 year), other implants, and absence of full text. Ultimately, 9 articles were included in the final analysis.

\section{Demographics}

A total of 115 patients from the 9 articles were included in the study, most of whom were female (Table 1). ${ }^{10,11,15-21)}$ Among the 9 included articles, 2 articles described 2 different implant
Search terms:

Distal humerus fracture,

hemiarthroplasty

Data sources:

PubMed, Ovid/MEDLINE,

Cochrane

*Less than 1 year mean

clinical follow-up,

full text unavailable,

other implant used

Table 1. Patient Demographics

\begin{tabular}{|c|c|c|c|c|c|c|c|}
\hline Year & Author & Level of study & Country & No. of case & Mean age (yr) & Male/Female & Mean F/U (mo) \\
\hline 2017 & Schultzel et al. ${ }^{15)}$ & IV & USA & 10 & $71.7 \pm 9.17$ & NA & 73.2 \\
\hline 2016 & Smith et al. ${ }^{16)}$ & IV & Australia & 3 & $39 \pm 11.7$ & NA & 44 \\
\hline 2015 & Phadnis et al. ${ }^{17)}$ & IV & UK & 16 & $78.7 \pm 8.06$ & $3 / 13$ & 35 \\
\hline 2015 & Nestorson et al. ${ }^{18)}$ & IV & Sweden & 42 & $67.36 \pm 17.36$ & $3 / 39$ & 34.29 \\
\hline 2015 & Heijink et al. ${ }^{19)}$ & IV & Netherland & 6 & $67.1 \pm 8.41$ & $0 / 6$ & 54 \\
\hline 2014 & Hohman et al. ${ }^{11)}$ & IV & USA & 7 & $63.42 \pm 14.7$ & $2 / 5$ & 36 \\
\hline 2013 & Smith and Hughes ${ }^{20)}$ & IV & Australia & 11 & $61.81 \pm 17.37$ & NA & 63.27 \\
\hline 2012 & Argintar et al..$^{21)}$ & IV & USA & 10 & $73.4 \pm 8.8$ & $1 / 9$ & 12 \\
\hline 2011 & Burkhart et al. $^{10)}$ & IV & Germany & 10 & $75.2 \pm 8.29$ & $0 / 10$ & 12.1 \\
\hline
\end{tabular}

Values are presented as number only, mean \pm standard deviation, or mean only.

F/U: follow-up, NA: not available.

Fig. 1. Flowchart of the search strategy in accordance with the Preferred Reporting Items for Systematic Reviews and Metaanalyses (PRISMA) guidelines. 
Table 2. Functional Scores and ROM Data from Included Studies

\begin{tabular}{|c|c|c|c|c|c|c|c|}
\hline Year & Author & No. of case & Mean F/U (mo) & $\operatorname{ROM}\left({ }^{\circ}\right)(\mathrm{FE})$ & ROM (PS) & MEPS & DASH score \\
\hline 2017 & Schultzel et al. ${ }^{15)}$ & 10 & 73.2 & $122.5 \pm 30.48$ & $135 \pm 17.15$ & $82.22 \pm 15.63$ & $32.07 \pm 26$ \\
\hline 2016 & Smith et al. ${ }^{16)}$ & 3 & 44 & $93.33 \pm 22.54$ & $121.6 \pm 96.73$ & $80 \pm 22.91$ & $14 \pm 10.81$ \\
\hline 2015 & Phadnis et al. ${ }^{17)}$ & 16 & 35 & $115.6 \pm 14.3$ & $171.5 \pm 8.7$ & $89.56 \pm 5.18$ & $11.23 \pm 5.77$ \\
\hline 2015 & Nestorson et al. ${ }^{18)}$ & 42 & 34.29 & $105.24 \pm 22.21$ & $178.29 \pm 5.43$ & $90 \pm 14.36$ & $20.26 \pm 16.63$ \\
\hline 2015 & Heijink et al. ${ }^{19)}$ & 6 & 54 & $95.8 \pm 16.5$ & $165 \pm 13.78$ & $78.33 \pm 25.43$ & $18.48 \pm 22.82$ \\
\hline 2014 & Hohman et al. ${ }^{11)}$ & 7 & 36 & $96.42 \pm 22.11$ & $160.7 \pm 13.7$ & $72.43 \pm 17.97$ & $33.42 \pm 25.15$ \\
\hline 2013 & Smith and Hughes ${ }^{20)}$ & 11 & 63.27 & $118.45 \pm 17.58$ & $159 \pm 52.58$ & $88.33 \pm 16.39$ & $20.9 \pm 19.6$ \\
\hline 2012 & Argintar et al. ${ }^{21)}$ & 10 & 12 & $102 \pm 25$ & $133.3 \pm 15$ & $77 \pm 16$ & $35.1 \pm 24$ \\
\hline 2011 & Burkhart et al. ${ }^{10)}$ & 10 & 12.1 & $107 \pm 15.49$ & $160 \pm 17.63$ & $91.3 \pm 11.49$ & $11.2 \pm 10.66$ \\
\hline
\end{tabular}

Values are presented as number only, mean only, or mean \pm standard deviation.

ROM: range of motion, F/U: follow-up, FE: flexion-extension, PS: pronation-supination, MEPS: Mayo elbow performance scores, DASH: Disabilities of the Arm, Shoulder and Hand.

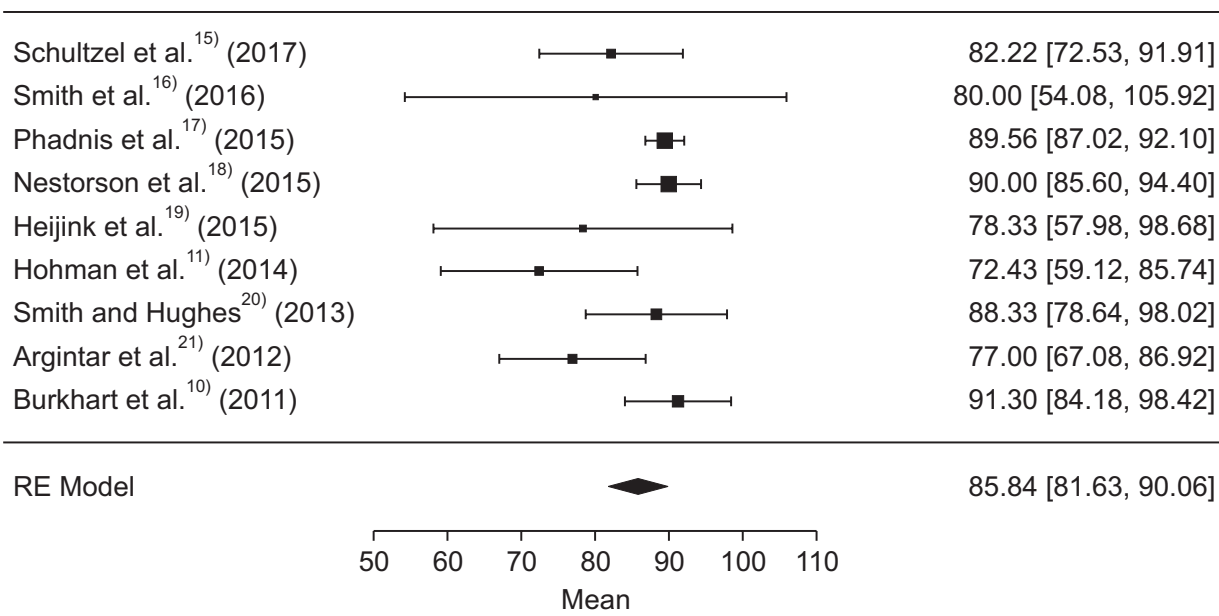

Test for heterogeneity: $\mathrm{Q}(\mathrm{df}=8)=15.4596, p=0.05$
Fig. 2. Clinical outcomes (Mayo elbow performance scores). df: degree of freedom.

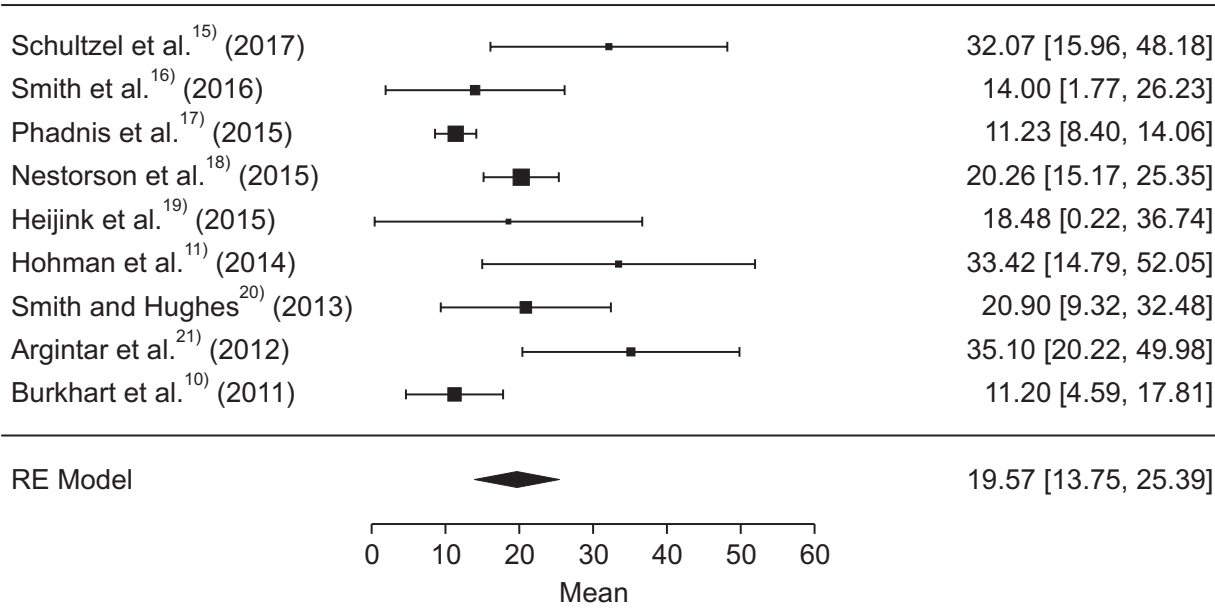

Test for heterogeneity: $\mathrm{Q}(\mathrm{df}=8)=28.2716, p=0.0004$
Fig. 3. Clinical outcomes (Disabilities of the Arm, Shoulder and Hand). df: degree of freedom. 
systems (the Sorbie-Questor system and the Latitude system); based on the raw data of these articles, our analysis included only those cases where the Latitude system had been used.

\section{Clinical Functional Scores}

Most studies reported good or excellent outcomes, flexionextension range of motion, and pronation-supination range of motion (Table 2). The weighted mean of Mayo elbow performance scores (MEPS) was $85.8\left(\mathrm{I}^{2}=60.36 \%\right.$; heterogeneity), and the weighted mean Disabilities of the Arm, Shoulder and Hand (DASH) score was $19.6\left(I^{2}=74.76 \%\right.$; heterogeneity) (Fig. 2, 3).

\section{Range of Motion}

The weighted mean flexion-extension and pronation-supination arcs were $107.6^{\circ}\left(I^{2}=57.24 \%\right.$; heterogeneity) and $157.5^{\circ}$ $\left(\mathrm{I}^{2}=95.58 \%\right.$; heterogeneity), respectively (Fig. 4, 5). Considering that arc of motion $100^{\circ}$ ( $30^{\circ}$ of extension to $130^{\circ}$ of flexion) and an arc of forearm rotation $100^{\circ}\left(50^{\circ}\right.$ of pronation to $50^{\circ}$ of supi- nation) are required for activities of daily living, $76 \%$ of patients achieved a functional range of motion for flexion and extension, and $99 \%$ achieved functional pronation and supination (Table 2).

\section{Complications}

Overall, there were 85 complications among the 115 patients evaluated. Cartilage wear $(n=45)$ was the most common complication reported, followed by heterotopic ossification $(n=39)$, loosening $(n=13)$, and neuropathy $(n=11)$. Other lesser complications included fracture (either intra- or postoperative) $(n=7)$, stiffness $(n=6)$, posterolateral rotatory instability $(n=4)$, continuous pain $(n=2)$, peri-prosthetic infection $(n=1)$ and wound breakdown $(n=1)$ (Table 3$)$.

\section{Discussion}

This systematic review focused solely on employing the Latitude system for hemiarthroplasty for distal humerus fracture. All

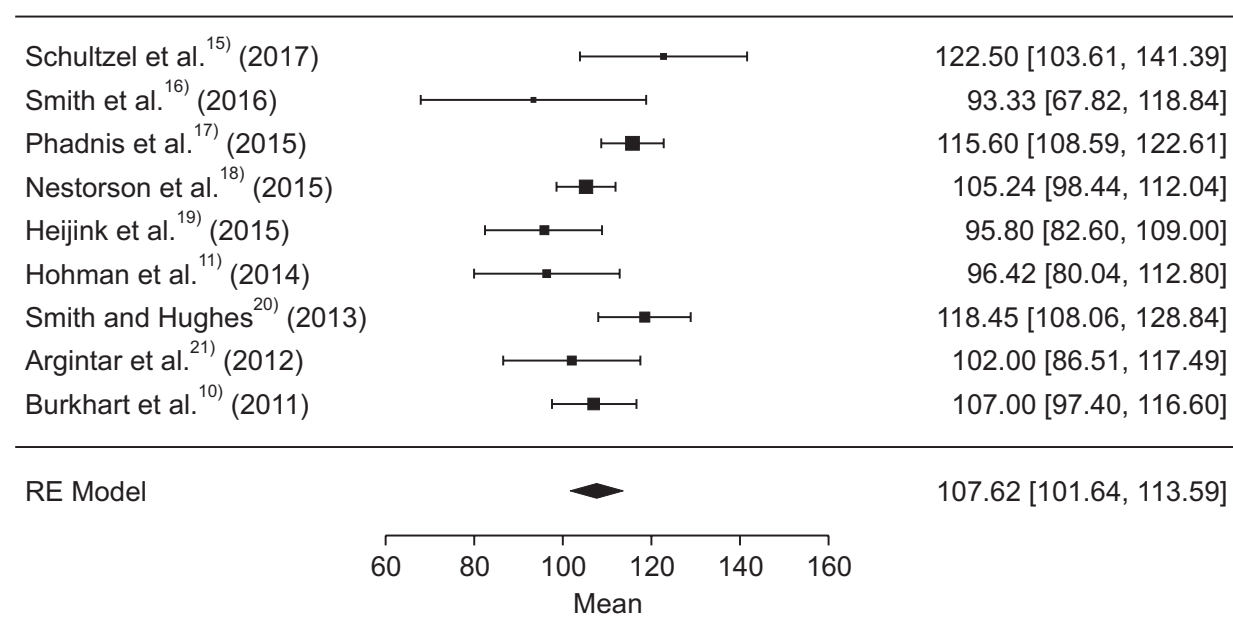

Test for heterogeneity: $\mathrm{Q}(\mathrm{df}=8)=18.2128, p=0.019$
Fig. 4. Range of motion (flexion-extension). df: degree of freedom.

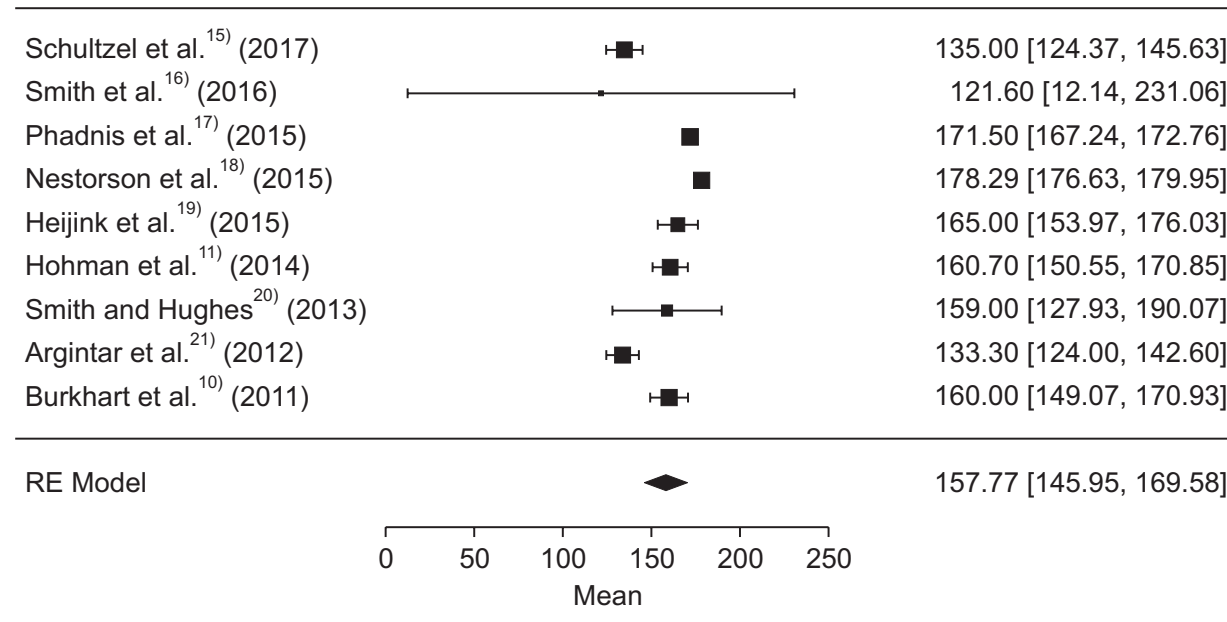

Test for heterogeneity: $\mathrm{Q}(\mathrm{df}=8)=168.9318, p<0.0001$
Fig. 5. Range of motion (supination-pronation). df: degree of freedom. 
Table 3. Associated Complications

\begin{tabular}{|c|c|c|}
\hline Year & Author & Complication (no. of case) \\
\hline 2017 & Schultzel et al. ${ }^{15)}$ & Intraoperative olecranon fracture (1), implant irritation (1) \\
\hline 2016 & Smith et al. ${ }^{16)}$ & Stiffness (1), aseptic loosening (2) \\
\hline 2015 & Phadnis et al. ${ }^{17)}$ & Ulnar nerve neurapraxia (1), radial head wear (3), ulnar wear (10), heterotopic ossifications (11) \\
\hline 2015 & Nestorson et al. ${ }^{18)}$ & $\begin{array}{l}\text { Limited range of movement (4), partial instability (1), loosening (1), sensory ulnar nerve symptoms (2), mild olecranon } \\
\text { wear (5) }\end{array}$ \\
\hline 2015 & Heijink et al. ${ }^{19)}$ & Some degree of instability (3), motoric and sensory sequelae of a partially recovered traumatic ulnar nerve lesion (1) \\
\hline 2014 & Hohman et al. ${ }^{11)}$ & $\begin{array}{l}\text { Postoperative ulnar neuropathy (1), intraoperative fracture of the humeral diaphysis (1), loosening (6), olecranon wear } \\
\text { mild (2) moderate (3) severe (2), radial head wear mild (5), moderate (2) }\end{array}$ \\
\hline 2013 & Smith and Hughes ${ }^{20)}$ & $\begin{array}{l}\text { Ulnar neuritis (4), stiffness (1), wound necrosis (1), ulnar wear (13), periprosthetic fractures (2), loosening (2), } \\
\text { heterotopic ossifications (13) }\end{array}$ \\
\hline 2012 & Argintar et al..$^{21)}$ & Symptomatic olecranon hardware (1), olecranon fracture intraoperatively after osteotomy (1), ulnar nerve numbness (1) \\
\hline 2011 & Burkhart et al. ${ }^{10)}$ & Triceps weakness (1), transient ulnar nerve irritation (1), superficial wound infection (1), heterotopic ossifications (2) \\
\hline
\end{tabular}

the studies included are classified as level IV evidence. The generally accepted optimal treatment for distal humerus fractures is ORIF, which is able to achieve stable fixation in majority of fractures; however, once attempts at fixation have failed, conversion to hemiarthroplasty avoids suboptimal fixation leading to poor outcomes. Distal humerus hemiarthroplasty is most typically used for intra-articular fractures of the distal humerus in geriatric populations. It also serves as a salvage procedure in younger patients with rheumatoid arthritis or tumors. To the best of our knowledge, by investigating the data of 115 patients who underwent distal humerus hemiarthroplasty, our work represents the most complete analysis for this specific indication.

Our analysis revealed that patients undergoing distal humerus hemiarthroplasty were more likely female and mostly in their seventh decade of age (mean, 66.41 years). Functional outcomes were summarized by means of 85.84 and 19.57 for MEPS and DASH scores, respectively. These outcomes are comparable with results associated with TEA. ${ }^{22)}$

Distal humerus hemiarthroplasty offers several benefits when compared to TEA, including preservation of the bone stock as well as minimization of postoperative restriction or revision surgery. Distal humerus hemiarthroplasty also preserves the native collateral ligaments, which helps preserve elbow proprioception. As for any other hemiarthroplasty, cartilage wear remains the most common challenge.

Despite the advantages of distal humerus hemiarthroplasty, complication rates remain higher than TEA. The most common complication in our review was cartilage wear (39.1\%), followed by heterotopic ossification (33\%), loosening $(11.3 \%)$, neuropathy (9.6\%), and stiffness (5.2\%). Others less reported complications were periprosthetic fracture $(4.34 \%)$, instability $(3.47 \%)$, wound necrosis $(1.73 \%)$, hardware irritation $(0.86 \%)$, and triceps muscle weakness $(0.86 \%)$. A common concern with any hemiarthroplasty is wearing of the native joint at the articulation point, resulting from a disparity in elasticity and surface roughness between the articular cartilage and prosthesis lining. With altered loading, the cartilage wears with time, eventually manifesting as and later causing instability and inducing the toggling movements. Loosening is also a worrisome and frequent complication of arthroplasty. We postulate that the cementing technique specifically contributes to this complication. In this situation, the prosthesis pullout is perpetuated by the pistoning movement of the cement mantle. However, the loosening rate of hemiarthroplasty was lower than that observed in TEA. ${ }^{22}$

The lack of standardized outcome measurements and consistent follow-up are limitations to our analysis. Published literature on distal humerus arthroplasty for distal humerus fracture is limited, and therefore our analysis lacks power. The case series that do exist are short, often with limited and irregular follow-up. We were unable to utilize certain statistical analysis methods, such as correlation testing, since the power of the test depends on the number of studies included. In a meta-analysis that includes less than 25 studies, the correlation test will be unable to withstand biases in the literature search.

This systematic review highlights the favorable outcomes of distal humerus arthroplasty for distal humerus fracture with the Latitude system. We propose that prospective analysis should be directed at forming regimented physical therapy to reduce complication rates.

\section{Conclusion}

Our study indicates that hemiarthroplasty is a viable option for comminuted distal humerus fracture. Satisfactory functional outcomes were observed in most patients. 


\section{References}

1. Mighell MA, Stephens B, Stone GP, Cottrell BJ. Distal humerus fractures: open reduction internal fixation. Hand Clin. 2015;31(4):591-604.

2. Nauth A, McKee MD, Ristevski B, Hall J, Schemitsch EH. Distal humeral fractures in adults. J Bone Joint Surg Am. 2011;93(7):686-700.

3. Sanchez-Sotelo J, Torchia ME, O'Driscoll SW. Complex distal humeral fractures: internal fixation with a principle-based parallel-plate technique. Surgical technique. J Bone Joint Surg Am. 2008;90 Suppl 2 Pt 1:31-46.

4. Ellwein A, Lill H, Voigt C, Wirtz P, Jensen G, Katthagen JC. Arthroplasty compared to internal fixation by locking plate osteosynthesis in comminuted fractures of the distal humerus. Int Orthop. 2015;39(4):747-54.

5. Sanchez-Sotelo J, Morrey BF. Total elbow arthroplasty. J Am Acad Orthop Surg. 2011;19(2):121-5.

6. Barco R, Streubel PN, Morrey BF, Sanchez-Sotelo J. Total elbow arthroplasty for distal humeral fractures: a ten-year-minimum follow-up study. J Bone Joint Surg Am. 2017;99(18):1524-31.

7. Harmer LS, Sanchez-Sotelo J. Total elbow arthroplasty for distal humerus fractures. Hand Clin. 2015;31(4):605-14.

8. Mellen RH, Phalen GS. Arthroplasty of the elbow by replacement of the distal portion of the humerus with an acrylic prosthesis. J Bone Joint Surg Am. 1947;29(2):348-53.

9. Adolfsson L, Hammer R. Elbow hemiarthroplasty for acute reconstruction of intraarticular distal humerus fractures: a preliminary report involving 4 patients. Acta Orthop. 2006;77(5):785-7.

10. Burkhart KJ, Nijs S, Mattyasovszky SG, et al. Distal humerus hemiarthroplasty of the elbow for comminuted distal humeral fractures in the elderly patient. J Trauma. 2011;71(3):635-42.

11. Hohman DW, Nodzo SR, Qvick LM, Duquin TR, Paterson PP. Hemiarthroplasty of the distal humerus for acute and chronic complex intra-articular injuries. J Shoulder Elbow Surg. 2014;23(2):265-72.
12. Rangarajan R, Papandrea RF, Cil A. Distal humeral hemiarthroplasty versus total elbow arthroplasty for acute distal humeral fractures. Orthopedics. 2017;40(1):13-23.

13. Moher D, Shamseer L, Clarke M, et al. Preferred reporting items for systematic review and meta-analysis protocols (PRISMA-P) 2015 statement. Syst Rev. 2015;4:1.

14. Jakobsen RB, Engebretsen L, Slauterbeck JR. An analysis of the quality of cartilage repair studies. J Bone Joint Surg Am. 2005;87(10):2232-9.

15. Schultzel M, Scheidt K, Klein CC, Narvy SJ, Lee BK, Itamura JM. Hemiarthroplasty for the treatment of distal humeral fractures: midterm clinical results. J Shoulder Elbow Surg. 2017;26(3):389-93.

16. Smith GC, Bayne G, Page R, Hughes JS. The clinical outcome and activity levels of patients under 55 years treated with distal humeral hemiarthroplasty for distal humeral fractures: minimum 2-year follow-up. Shoulder Elbow. 2016;8(4):264-70.

17. Phadnis J, Banerjee S, Watts AC, Little N, Hearnden A, Patel VR. Elbow hemiarthroplasty using a "triceps-on" approach for the management of acute distal humeral fractures. J Shoulder Elbow Surg. 2015;24(8):1178-86.

18. Nestorson J, Ekholm C, Etzner M, Adolfsson L. Hemiarthroplasty for irreparable distal humeral fractures: medium-term follow-up of 42 patients. Bone Joint J. 2015;97(10):1377-84.

19. Heijink A, Wagener ML, de Vos MJ, Eygendaal D. Distal humerus prosthetic hemiarthroplasty: midterm results. Strategies Trauma Limb Reconstr. 2015;10(2):101-8.

20. Smith GC, Hughes JS. Unreconstructable acute distal humeral fractures and their sequelae treated with distal humeral hemiarthroplasty: a two-year to eleven-year follow-up. J Shoulder Elbow Surg. 2013;22(12):1710-23.

21. Argintar E, Berry M, Narvy SJ, Kramer J, Omid R, Itamura JM. Hemiarthroplasty for the treatment of distal humerus fractures: short-term clinical results. Orthopedics. 2012;35(12):1042-5.

22. Kamineni S, Morrey BF. Distal humeral fractures treated with noncustom total elbow replacement. J Bone Joint Surg Am. 2004;86(5):940-7. 